\title{
Z-Map Based Cutting Force Prediction for Elliptical Ultrasonic Vibration-Assisted Milling Process
}

\section{Zhongqun Li ( $\nabla$ zhqunli@163.com )}

Hunan University of Technology https://orcid.org/0000-0001-6922-1483

Jiandong Xiao Xiao

Hunan University of Technology

\section{Xiong HAN}

AVIC Chengdu Aircraft Industrial (Group) Co., Ltd., Chengdu

\section{Weifeng ZHANG}

Hunan University of Technology

\section{Research Article}

Keywords: Elliptical ultrasonic vibration-assisted milling, Cutting force prediction, Separating characteristics, Z-map model

Posted Date: November 16th, 2021

DOI: https://doi.org/10.21203/rs.3.rs-1059092/v1

License: (c) (1) This work is licensed under a Creative Commons Attribution 4.0 International License. Read Full License

Version of Record: A version of this preprint was published at The International Journal of Advanced Manufacturing Technology on March 3rd, 2022. See the published version at https://doi.org/10.1007/s00170-022-08976-w. 


\title{
Z-map Based Cutting Force Prediction for Elliptical Ultrasonic Vibration-assisted Milling Process
}

\author{
Zhongqun $\mathrm{Li}^{* 1,3}$, Jiandong Xiao ${ }^{1}$, Xiong $\mathrm{Han}^{2}$, Weifeng Zhang ${ }^{1}$ \\ 1School of Mechanical Engineering, Hunan University of Technology, Zhuzhou, 412007, China \\ ${ }^{2}$ AVIC Chengdu Aircraft Industrial (Group) Co., Ltd., Chengdu 610091, China \\ ${ }^{3}$ Jiangxi Research Institute, Beihang University, Nanchang, 330224, China \\ *Corresponding author: zhqunli@163.com
}

\begin{abstract}
Elliptical ultrasonic vibration-assisted milling (EUVAM) adds high-frequency vibration to conventional milling (CM) to realize high-frequency intermittent milling. It has broad application prospects in the processing of difficultto-cut materials such as titanium alloys, superalloys and hard and brittle materials. To reveal the mechanism of the highly intermittent cutting nature in EUVAM, according to the motion relationship between cutting edge and workpiece and the Z-map model of the workpiece, a method and its algorithm for calculating undeformed cutting thickness and thus the cutting force in EUVAM are proposed. The simulation results show that EUVAM can improve the actual cutting speed when compared with CM, and the proportion of idle cutting time will directly determine the intermittent degree of the milling process. The experiment of EUVAM is performed to verify the correctness of the proposed cutting force model, and the impact of spindle speed on the cutting force in EUVAM is also analyzed.
\end{abstract}

Keywords: Elliptical ultrasonic vibration-assisted milling; Cutting force prediction; Separating characteristics; Zmap model;

\section{Introduction}

Elliptical ultrasonic vibration-assisted cutting technology (EUVAC) is a new machining method that is based on the combination of traditional cutting methods and ultrasonic vibration machining technology, that is, by improving the structure and function of traditional machine tools, ultrasonic vibration is applied to tools or workpieces, and the ultrasonic vibration energy is added to the machining process to further enhance its machining performance [1]. On the basis of one-dimensional ultrasonic vibrationassisted cutting, EUVAM and its cutting principle were first proposed and revealed by Shamoto and Moriwaki [2], which is a cutting process where a cutting tool moves along an elliptical path generated by adding the cyclic vibrations on the cutting tool in the cutting and chip flow directions.

When elliptical ultrasonic vibration machining technology is combined with the conventional milling technology, it is called EUVAM. Milling is an intermittent cutting process since each cutting tooth touches the workpiece periodically within the range of entry and the exit angle at the tooth-passing frequency. The externally applied elliptical ultrasonic vibrations cause additional, high-frequency toolworkpiece contact and separation, which changes the kinematics and dynamics of milling process. It has been demonstrated that EUVAM can reduce cutting forces and heat generation, which improves the surface finish and the cutting tool life. As a result, the EUVAM is especially suitable for precision machining of hardened steel molds with mirror surface finish [2], carbon fiber reinforced plastic (CFRP) parts in the aviation industry to avoid delamination [3], and machining of titanium alloys to achieve longer tool life [4].

In order to achieve high-efficiency chatter-free EUVAM, the dynamics of the vibration-assisted milling process should be modeled accurately. Although the stability of conventional milling operations has been studied extensively in the literatures $[5,6]$, the stability of milling under ultrasonic vibrations has not been investigated sufficiently. Xiao et al. [7] numerically and experimentally studied the chatter 
stability of a 1-DOF vibration-assisted turning process and reported achieving a larger depth of cut, but they did not predict stability lobes of the process. Ma et al. [8] excited elliptical ultrasonic vibration on a turning tool and analyzed the chatter stability in the frequency domain using the Nyquist criteria. They considered the periodic contact time of the actuator by Fourier Series and used the dynamic orthogonal cutting model in the stability solution. Wan et al. [9] reported the first analytical chatter stability of the vibration-assisted milling process using the semi-discrete method. In his study, the vibration was excited along the feed or normal direction from the workpiece side, and the high-frequency tool-workpiece separations changed the time delay dynamically and hence shifted the stability lobes. Gao and Altintas 10] proposed a dynamic model of the synchronized elliptical ultrasonic vibration-assisted milling, solved it using the semi-discrete time-domain method, and validated the predicted stability diagram experimentally.

Cutting force is a significant factor affecting the milling quality and efficiency, especially when machining the nickel alloy, titanium alloy, and composite parts with complex shapes and thin walls. In order to reveal the influence of EUVAM on cutting force and surface integrity, systematic researches have been conducted using numerical simulation or experimental methods. Using the 3D finite element method, Muhammed et al. [11] compared the cutting force between ultrasonic vibration cutting and conventional cutting and considered that the vibration cutting can reduce cutting force due to the increase of cutting speed and decrease of tool-workpiece engagement. Tong et al. [12] using both finite element method and experimental method to investigate the transient cutting processes of titanium alloy with EUVAC method, compared the main cutting force of EUVAC with that of convention cutting (CC) and discovered that under EUVAC, the reduction rate of cutting forces decreases with the increase in cutting depth, and increases with the increase in cutting speed. Based on the kinematic characteristics of toolworkpiece and the separation criteria of intermittent cutting condition during high-speed EUVAM, Liu et al. [13] established a chip thickness model and the tool dynamic cutting relief angle model and investigated the effects of vibration amplitude on cutting force and surface integrity. Abootorabi et al. [14] experimentally studied the effects of cutting speed and workpiece vibration amplitude on the cutting force. Results showed that as the cutting speed increases, the effect of ultrasonic vibration on the milling process decreases and the cutting force in the EUVAM and CM processes become closer to each other. Han et al. [15] introduced a velocity coefficient $K$ to describe the separating characteristic of the EUVAM and theoretically analyzed the separating type and non-separating type cutting using the EUVAM method. Theoretical analysis and experimental results show that compared with non-separating EUVAM, the separating one can bring more reduction of the average radial cutting force.

As mentioned above, although there are many studies on the cutting force of EUVAM, most of them only focus on the characteristics or performance of EUVAM, lacking mechanism analysis of its change, and cannot reveal the changes of instantaneous chip thickness and dynamic cutting force with cutting conditions. Even if there are a few studies using analytical methods to calculate the undeformed cutting thickness and thus to predict the cutting forces in EUVAM [9]. However, due to the complexity of elliptical ultrasonic vibration, the cutting edges may lose contact with the workpiece at a high frequency during single tooth-passing period. Simply expressing the undeformed cutting thickness as the difference between the current tooth $j$ position $\left(p_{j}(t)\right)$ at time $t$ and the previous in-cut tooth $q$ in the radial direction with $\tau$ delay $\left(p_{q}(t-\tau)\right.$ cannot accurately reflect the real situation of EUVAM, and of course, it cannot accurately predict the undeformed cutting thickness. The Z-map model was first proposed by Lin et al. in which the entity is first projected on the XY plane and then meshed according to the required simulation accuracy. In terms of data structure, the Z-map model records the height of each grid point in 
the $\mathrm{Z}$ direction on the XY plane, so the Z-map model uses a two-dimensional array to record the height of the three-dimensional model. Therefore, the Z-map model is an ideal data structure for three-axis NC milling simulation $[16,17]$. In this paper, the Z-map model is used to represent the workpiece. The instantaneous undeformed cutting thickness is obtained by calculating the intersection between the cutting edges and the workpiece at each discrete time. The instantaneous cutting force is obtained by applying the instantaneous rigid cutting force model.

The paper is organized into six sections. In section 2, based on the kinematic modeling of EUVAM, the impact of spindle speed on the cutting speed in both EUVAM and CM is analyzed. Section 3 gives the methodology and algorithm of undeformed cutting thickness in EUVAM based on the Z-map model. Section 4 establishes the ultrasonic milling force model by applying the instantaneous rigid force model to each cutting-edge element. In section 5 the elliptical ultrasonic vibration device is designed and tested, the predicted cutting force of EUVAM is compared with the experimental result, and the influence of spindle speed on the ultrasonic milling force is analyzed. Some conclusions are drawn in Section 6.

\section{Kinematic modeling of EUVAM}

\subsection{Tooltip motion equation}

When two resonant vibrations of certain frequency and amplitude are applied to the tool holder, the $2 \mathrm{D}$ vibrations with a $90^{\circ}$ phase shift are in a plane perpendicular to the tool axis and tangential to the cutting speed direction, forming the elliptical vibration. As shown in Fig. 1, due to the existence of elliptical ultrasonic vibration of the cutting tool, the motion of the tooltip is very complex. It consists of the rotational motion of the cutting tool around the spindle axis, the elliptical vibration around the tool axis, and the linear feed motion of the cutting tool.

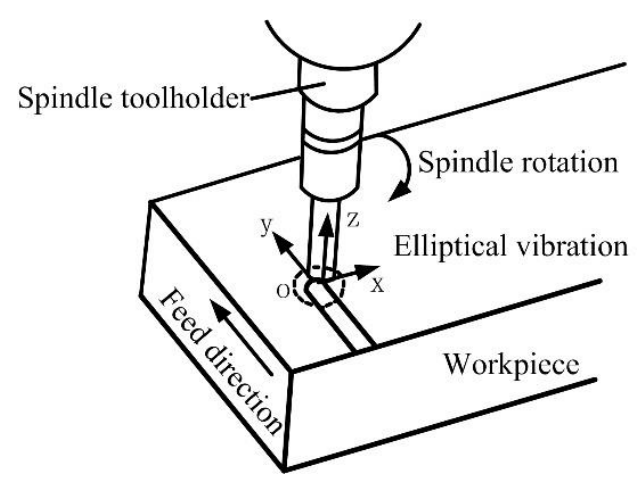

Fig. 1 Schematic illustration of tooltip motion in EUVAM

The feed motion of the cutting tool can be expressed as:

$$
\left\{\begin{array}{l}
x_{f}=0 \\
y_{f}=v_{f} t \\
\quad v_{f}=f_{t} n N / 60
\end{array}\right.
$$

where $v_{f}$ is the feed rate, $f_{t}$ is the feed fate per tooth, $n$ is the spindle speed, $N$ is the teeth number, $t$ is the time. The cutting tool rotation around the spindle axis can be expressed as follows:

$$
\left\{\begin{array}{l}
x_{r}=R \cos (\omega t) \\
y_{r}=R \sin (\omega t) \\
\omega=\pi n / 30
\end{array}\right.
$$

where $R$ is the radius of the cutting tool and $\omega$ is the angular velocity of the cutting tool.

As shown in fig. 2, the cutting edge performs elliptical ultrasonic vibration in the Oxy plane. Suppose that the initial position of the long axis of the ellipse is in the $\mathrm{x}$-axis direction, and the rotation movement of the cutting tool around the spindle axis makes the vibration direction of the tooltip ellipse 
constantly change. When the cutting tool rotates $\alpha$, the direction of the long axis of the ellipse is consistent with the direction of the $U$-axis in the $O u v$ coordinate system. The motion equation of elliptical vibration can be expressed as:

$$
\left\{\begin{array}{l}
u=0.5 a \cos (2 \pi f t) \\
v=0.5 b \sin (2 \pi f t)
\end{array}\right.
$$

where $a$ is the amplitude in the feed direction, $b$ is the amplitude perpendicular to the feed direction, $f$ is the vibration frequency.
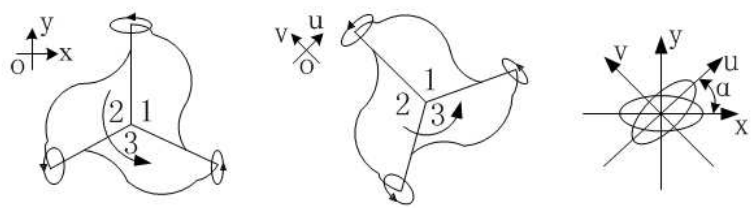

Fig.2 Variation of the elliptical vibration trajectory with rotation of the cutting tool

When the ellipse equations in $O u v$ are transformed into the trajectory equations in $O x y$, the coordinate transformation can be expressed as:

$$
\left\{\begin{array}{l}
x_{e}=u \cos (\omega t)-v \sin (\omega t) \\
y_{e}=u \sin (\omega t)+v \cos (\omega t)
\end{array}\right.
$$

Considering the above three parts of motion, the motion equation of the tooltip can be obtained as follows:

$$
\left\{\begin{array}{c}
x=R \cos (\omega t)+u \cos (\omega t)-v \sin (\omega t) \\
y=v_{f} t+R \sin (\omega t)+u \sin (\omega t)+v \cos (\omega t)
\end{array}\right.
$$

According to Eq. (5), the tooltip trajectory in EUVAM can be as shown in Fig.3. It can be seen from the figure that the tooltip trajectory in EUVAM is generally similar to that in CM (its main motion trajectory is approximately an arc), except some elliptical vibration trajectories exist in EUVAM. Different cutting conditions lead to two completely different cutting statuses. Fig.3b shows the situation that when the rotation of the cutting tool is dominant, although the elliptical vibration makes the tooltip not move in an approximately circular motion, the cutting tooth loses contact with the workpiece only once within one tooth-passing period. Fig. $3 \mathrm{C}$ shows that when the elliptical vibration is dominant, the elliptical vibration not only makes the tooltip no longer make an approximately circular motion, but also makes the cutting teeth separate from the workpiece many times within one tooth-passing period. When the elliptical vibration is dominant, points A, B, C, D, E correspond to an ultrasonic vibration period. The cycle can be divided into 4 stages: A-B is the empty cutting stage, the tooth moves towards the workpiece at a high speed, and the cutting force is zero; B-C is the cutting-in stage, and the cutting thickness increases gradually; C-D is the cutting-out stage, and the cutting thickness decreases gradually; D-E is the empty cutting stage. The tooth cuts out of the workpiece from point $\mathrm{D}$, separates from the workpiece, and the cutting force remains zero.
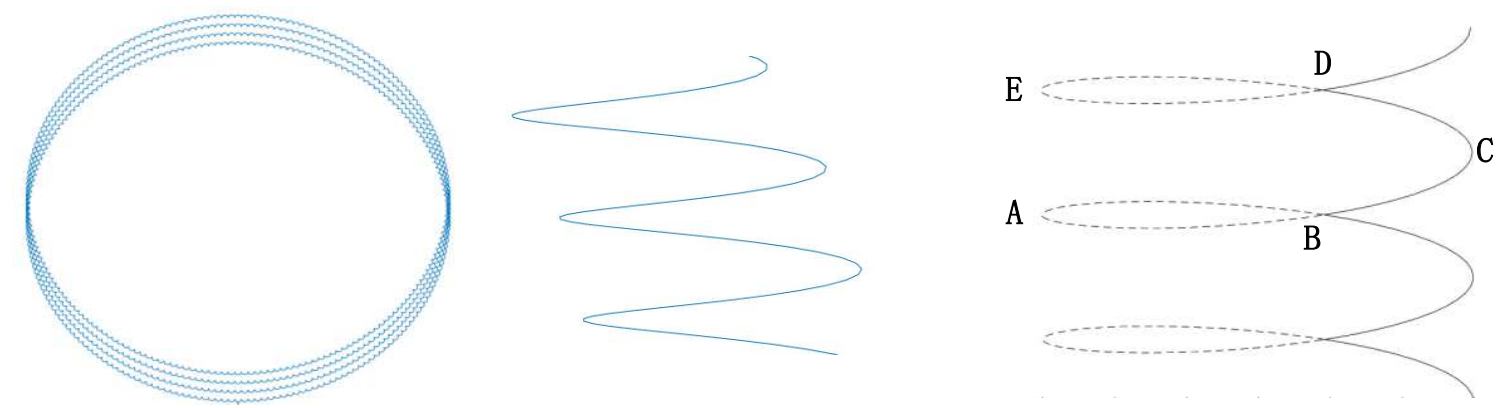


\subsection{Tooltip velocity equation}

Fig. 3 Tooth trajectory in EUVAM

Find the derivative of Eq. (5), the velocity equation of tooltip can be obtained as follows:

$$
\left\{\begin{array}{c}
v_{x}=-R \omega \sin (\omega t)+u^{\prime} \cos (\omega t)-u \omega \sin (\omega t)-v^{\prime} \sin (\omega t)-v \omega \cos (\omega t) \\
v_{y}=v_{f}+R \omega \cos (\omega t)+u^{\prime} \sin (\omega t)+u \omega \cos (\omega t)+v^{\prime} \cos (\omega t)-v \omega \sin (\omega t)
\end{array}\right.
$$

The synthesis velocity $(v)$ and its direction $(\alpha)$ are:

$$
\left\{\begin{array}{c}
v=\sqrt{v_{x}^{2}+v_{y}^{2}} \\
a=\tan ^{-1} v_{y} / v_{x}
\end{array}\right.
$$

The tangential and radial cutting speed are:

$$
\left\{\begin{array}{l}
v_{t}=-v_{x} \sin (\omega t)+v_{y} \cos (\omega t) \\
v_{r}=v_{x} \cos (\omega t)+v_{y} \cos (\omega t)
\end{array}\right.
$$

According to Eq. (6)-Eq. (8), the tangential cutting speed is predicted by changing the spindle speed, the comparison of tangential cutting speed between EUVAM and CM at different spindle speed is shown in Fig. 5. The figure shows that due to the participation of elliptical ultrasonic vibration, the tangential cutting speed in EUVAM fluctuates around that in CM; when the spindle speed is high, the minimal tangential cutting speed is always greater than zero, that is, during one tooth-passing period, the tooth keeps cutting the workpiece until it exceeds the exit angle. Under this situation, although the tangential cutting speed fluctuates, it is still performing the conventional milling in essential, which can be supported by the tooltip trajectory shown in Fig. 3b. With the decrease of the spindle speed, the minimal tangential cutting speed will cross the zero line, that is, the tooth will not cut the workpiece forward, but retreat backward. The phenomenon is bound to cause the tooth to lose contact with the workpiece, resulting in a temporary interruption of the milling process and brief cooling of the cutting zone, which is also the purpose and advantage of EUVAM. In Fig.4b, the minimal tangential cutting speed is just zero; in Fig.4c, the time tangential cutting speed is negative accounts for about $1 / 4$ of the whole vibration period; in Fig.4d, with the further decrease of the spindle speed, the time when the tangential cutting speed is negative, accounting for about $1 / 3$ of the whole vibration period. In the whole vibration period, the optimal proportion of idle cutting time remains to be determined.

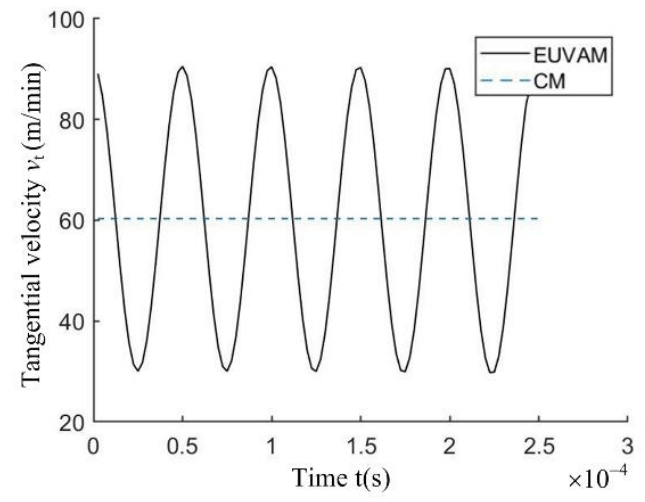

a. $n=2400 \mathrm{r} / \mathrm{min}$

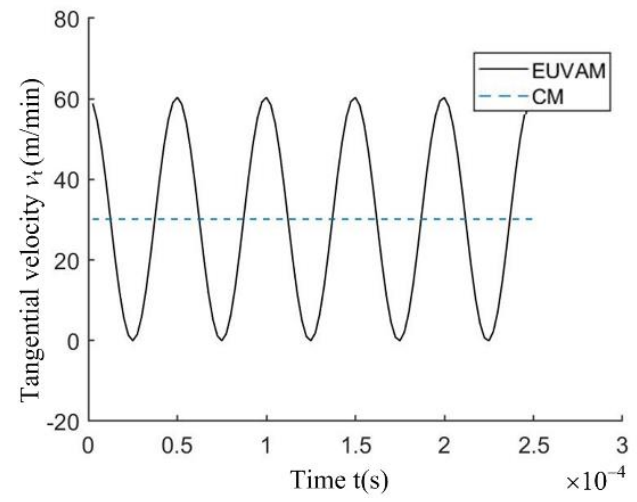

b. $n=1200 \mathrm{r} / \mathrm{min}$ 

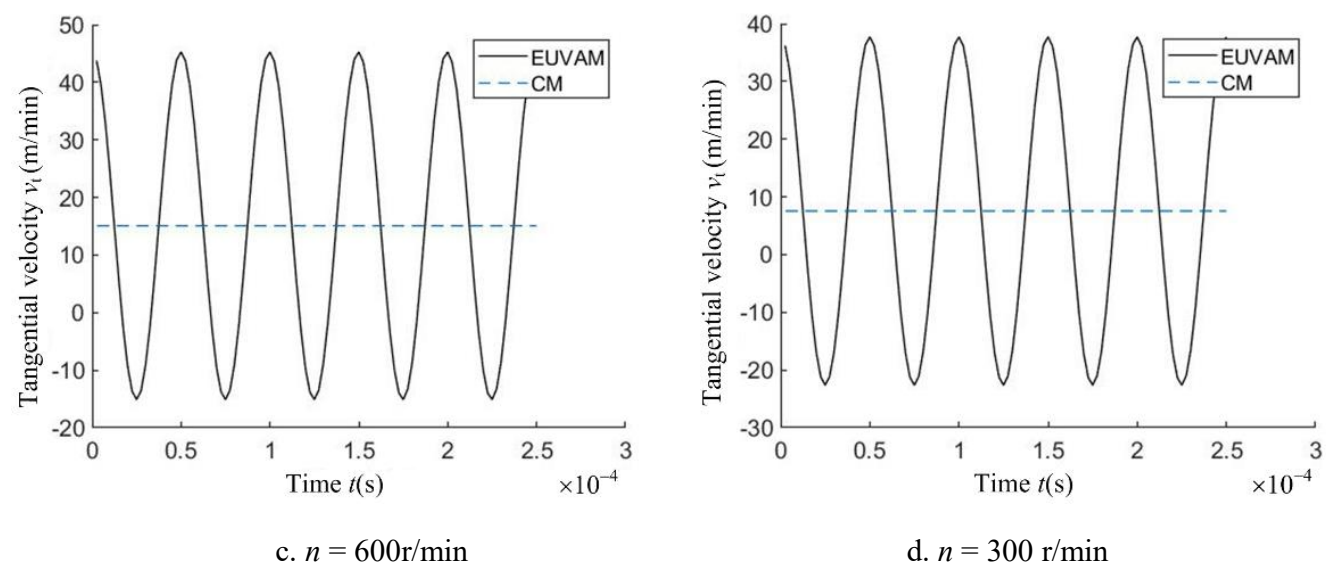

Fig. 4 Comparison of tangential cutting speed between EUVAM and CM under different spindle speed ( $a=0.012 \mathrm{~mm}, b=0.008 \mathrm{~mm}, f=20000 \mathrm{~Hz}, R=4 \mathrm{~mm}, f_{z}=0.1 \mathrm{~mm} /$ tooth,$\left.N=2\right)$

Fig. 5 shows the change of tooltip cutting speed in two typical EUVAM. As shown in the figure, regardless of the spindle speed, the maximal cutting speed in EUVAM is much higher than that in CM. It can also be seen from Fig.3-Fig.5, during one elliptical vibration cycle, B-C-D is the cutting section. When the tooltip moves to point $\mathrm{C}$, the ultrasonic vibration speed direction is consistent with the tooltip rotation speed direction, and its synthesis cutting speed reaches the maximum. When the tooltip moves to point $\mathrm{D}$, the synthesis cutting speed reaches the minimum. As the spindle speed $n$ is $600 \mathrm{r} / \mathrm{min}$, the maximal cutting speed is $49.48 \mathrm{~m} / \mathrm{min}$, the minimal cutting speed is $15.10 \mathrm{~m} / \mathrm{min}$, both are greater than the cutting speed in CM $(15.09 \mathrm{~m} / \mathrm{min})$. As the spindle speed $n$ is $300 \mathrm{r} / \mathrm{min}$, the maximal cutting speed is $46.20 \mathrm{~m} / \mathrm{min}$, the minimal cutting speed is $22.60 \mathrm{~m} / \mathrm{min}$, both are greater than the cutting speed in CM (7.54 $\mathrm{m} / \mathrm{min})$.

It can be seen from the above analysis, the cutting speed in EUVAM is greater than that in CM, the cutting speed near point $\mathrm{C}$ reaches the speed required for high-speed milling of titanium alloy or superalloy. The high-speed milling section accounts for $1 / 5$ of the elliptical vibration period. Therefore, the adoption of EUVAM can realize high-speed milling to some extent at a relatively low spindle speed. If the amplitude and frequency can be further improved, the effect of high-speed milling will be more obvious.

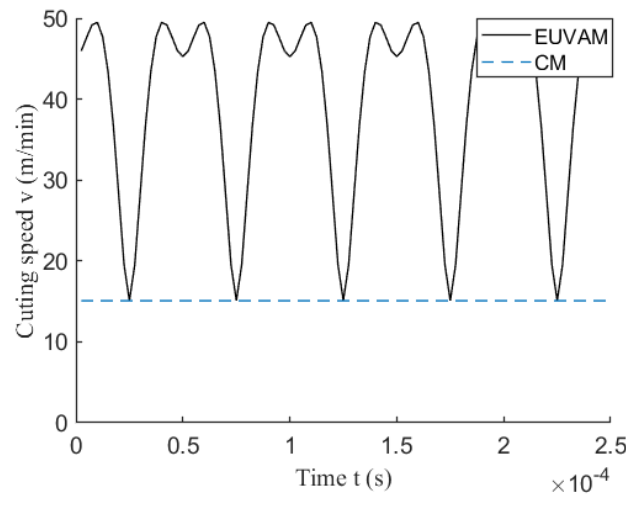

a. $n=600 \mathrm{r} / \mathrm{min}$

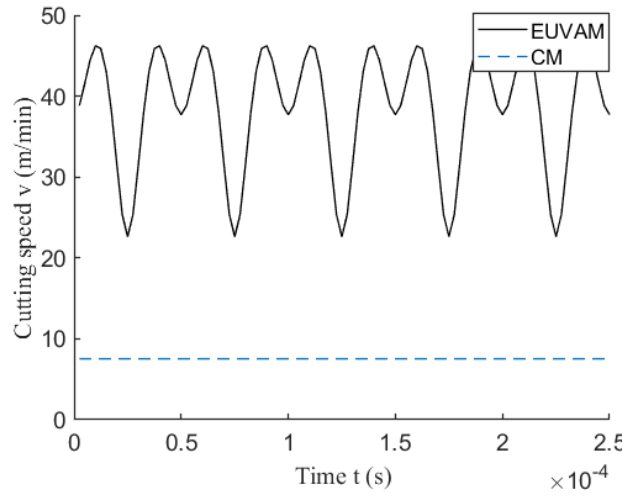

b. $n=300 \mathrm{r} / \mathrm{min}$

Fig. 5 Cutting speed curve in EUVAM under different spindle speed ( $a=0.012 \mathrm{~mm}, b=0.008 \mathrm{~mm}, f=20000 \mathrm{~Hz}, R=4 \mathrm{~mm}, f_{z}=0.1 \mathrm{~mm} /$ tooth, $N=2$ )

\section{Calculation of undeformed cutting thickness}

\subsection{Z-map model of the workpiece}


So far, the most widely used approach for calculating the undeformed cutting thickness was proposed by Tlusty et al., in which the trajectory of the cutting tool is approximately regarded as an arc. Suppose the feed rate per tooth is $f_{t}$, when the rotation angle of the cutting tool is $\phi$, the undeformed cutting thickness can be approximately expressed as:

$$
h=f_{t} \sin \theta
$$

However, during the process of ultrasonic vibration milling, due to the comprehensive action of tool rotation and elliptical ultrasonic vibration, the path of cutting tooth becomes very complex (see Fig. 3 ), which makes the above equation is no longer applicable to the elliptical ultrasonic milling process. Due to the existence of elliptical ultrasonic vibration, the cutting tooth often jumps away from the workpiece, and the its motion trajectory is not consistent with the workpiece contour. The value obtained from the difference between the current tooth position and the previous tooth position in the radial direction with $\tau$ delay is not always the undeformed cutting thickness. As shown in Fig.6, $O_{\mathrm{p}}(t)$ is the origin of the workpiece coordinate system, $O_{t}(t)$ is the tool center position which is affected by the feed rate, the elliptical vibration amplitude, and frequency, $P(t)$ and $Q(t)$ are the current tooth position and the intersection point of the machined surface with the cutting tool in the radial direction of the cutting tooth respectively, $h(t)$ is the undeformed cutting thickness in the radial direction. Therefore, it is necessary to find other methodologies to calculate the undeformed cutting thickness. In the following, a Z-map model is used to represent the surface of the workpiece to obtain the undeformed cutting thickness.

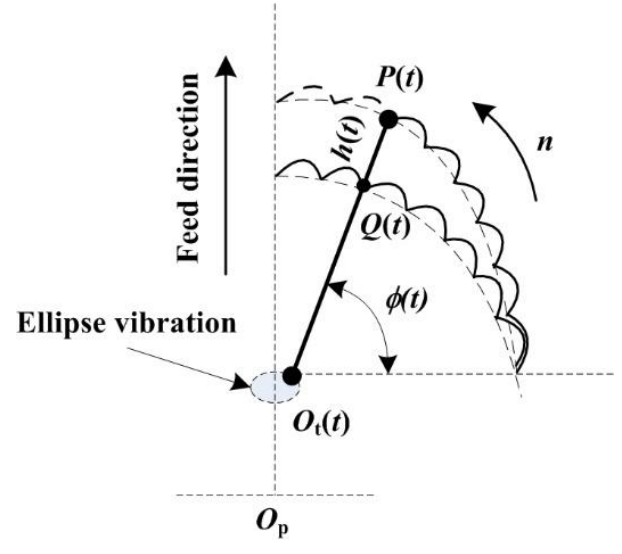

Fig.6 Static chip thickness for EUVAM

The Z-map representation of the workpiece for EUVAM is shown in Fig.7, $O_{i}$ and $P_{i}$ are the tool center and the tooth position at time $t, O_{\mathrm{i}+1}$ and $P_{i+1}$ are the tool center and the tooth position at time $t+\mathrm{d} t$, $R$ is the radius of the cutting tool, $\mathrm{d} x$ and $\mathrm{d} y$ are the grid size in $x$ and $y$ direction, symbol "o" represents the grid in the unmachined surface, symbol "•" represents the grid in the surface to be machined during period $\mathrm{d} t$, and the blank indicates the grid in the machined surface. 


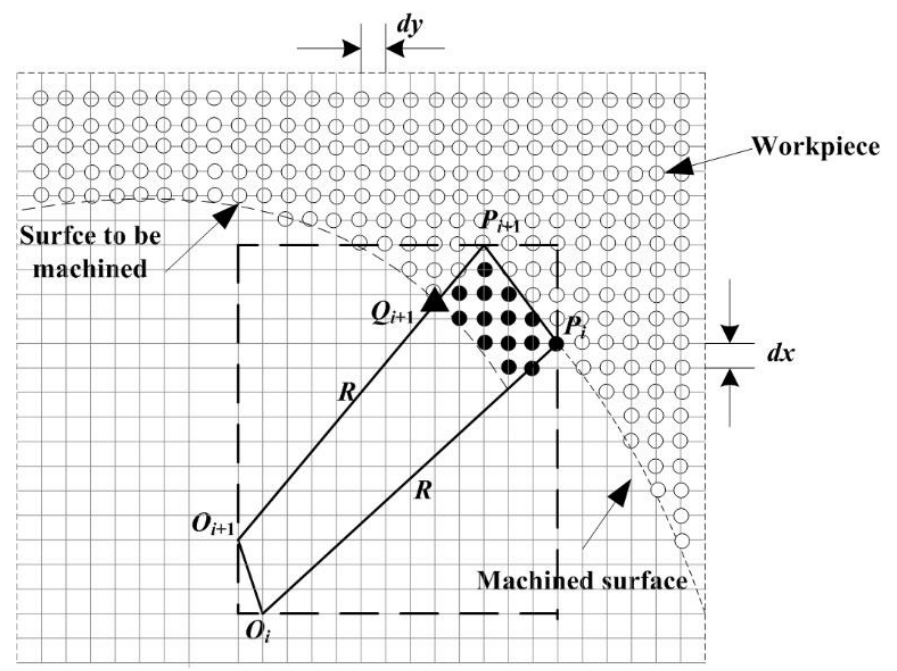

Fig. 7 Z-map representation of the workpiece for EUVAM

\subsection{Methodology and algorithm for obtaining the undeformed cutting thickness}

The methodology and algorithm used for calculating the undeformed cutting thickness based on the z-map model are shown in Fig.8, and the basic procedures are as follows:

1) Step 1: Set the appropriate grid size $\mathrm{d} x, \mathrm{~d} y$ to ensure the accuracy of the Z-map model, and make $\mathrm{Z}\left(1: x_{\text {num }}, 1: y_{\text {num }}\right)=a_{p}$ to initialize the Z-map model of the workpiece.

2) Step 2: Set the appropriate time increment $\mathrm{d} t$ to realize the discretization of the elliptical ultrasonic vibration milling process.

3) Step 3: For each discrete time $t$, calculate the coordinates of the tool center position $O_{\mathrm{i}+1}$ and the current tooth point $P_{\mathrm{i}+1}$ according to Eq. (4)-Eq. (5).

4) Step 4: Using the coordinates of $O_{\mathrm{i}}, O_{\mathrm{i}+1}, P_{\mathrm{i}}, P_{\mathrm{i}+1}$ to obtain the rectangle which is represented by $\left(x_{\min }, y_{\min }, x_{\max }, y_{\max }\right.$ ) (see the dashed line in the figure), and mesh this rectangle with $\mathrm{d} x$ and $d \mathrm{y}$.

5) Step 5: Use the binary search method to obtain the intersection point $Q_{i+1}$ between the cutting edge and the workpiece located at the straight line $O_{i+1} P_{i+1}$, and then obtain the instantaneous undeformed cutting thickness.

6) Step 6: For each grid within the rectangle, use the library function inploygon in Matlab to judge whether a specified point is inside the quadrilateral $O_{i} P_{i} P_{i+1} O_{i+1}$, if $Z(j, k)=a_{p}$ and if the grid is inside the quadrilateral, update the grid by setting $Z(j, k)=0$.

7) Repeat step 3 to step6 until the end of the simulation.

\subsection{Simulation and analysis of undeformed cutting thickness in EUVAM}

Using the above-mentioned approaches, the undeformed cutting thickness of each tooth in EUVAM at any angle can be obtained. The simulation results under different cutting conditions are shown in Fig.9.

It is not difficult to understand from the figure that the undeformed cutting thickness in EUVAM is no longer regular as that in $\mathrm{CM}$, but an irregular curve varying with the frequency and amplitude of the elliptical vibration and the spindle speed. When the spindle speed is high, as shown in Fig. 9a and Fig. $9 \mathrm{~b}$, because the rotation of the cutting tool is dominant, although the elliptical ultrasonic vibration will make the undeformed cutting thickness change sharply, it will not cause the cutting tooth to leave the cutting area only when the cutting tool starts to cut into the workpiece, will there be a short period of high intermittent cutting process. When the spindle speed is low, as shown in Fig. 9c and Fig. 9d, because the elliptical vibration is dominant, it will cause the cutting tooth to leave the cutting zone at a high frequency and make the deformed cutting thickness zero. At this time, the cutting edge of the current tooth loses contact with the workpiece and idle cutting occurs. With the further decrease of rotation speed, 
the proportion of idle cutting time in the overall cutting time increases. Therefore, it is not difficult to infer that elliptical ultrasonic vibration milling at this time is a highly intermittent cutting process. The high-frequency intermittent contact and separation between the cutting tool and the workpiece help the cutting fluid enter the cutting area and cool the cutting tooth, which helps to improve the tool life and the surface quality of the workpiece.

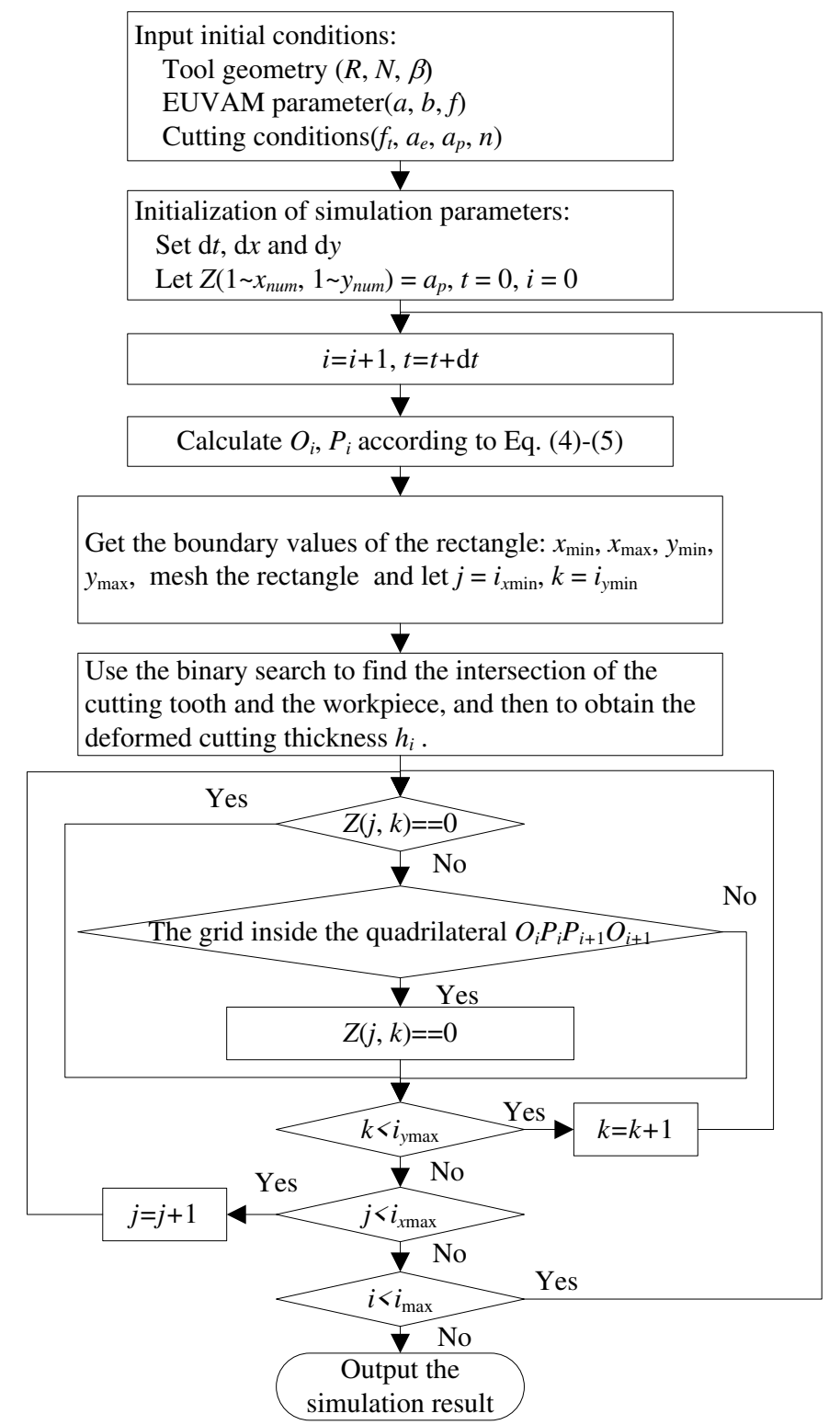

Fig. 8 Flow chart for calculating the undeformed cutting thickness based on z-map model 


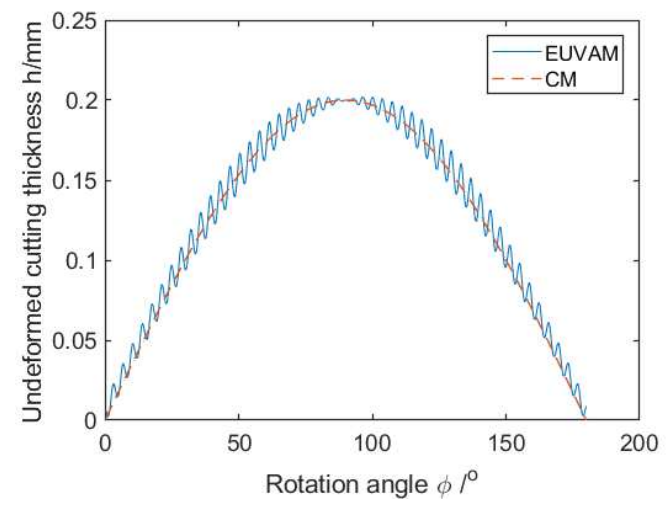

a. $n=12000 \mathrm{r} / \mathrm{min}$

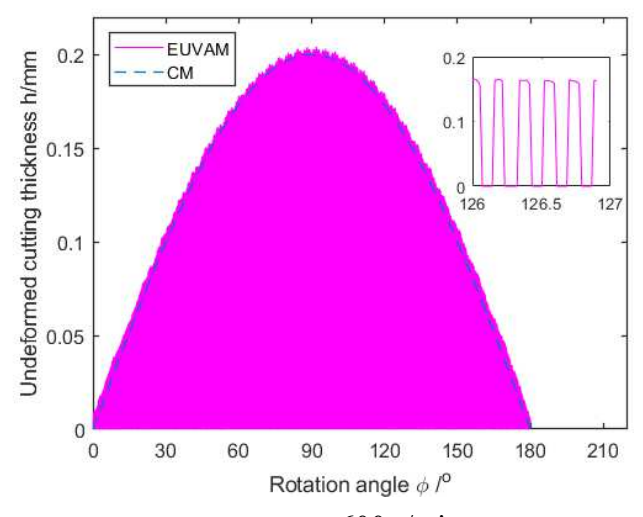

c. $n=600 \mathrm{r} / \mathrm{min}$

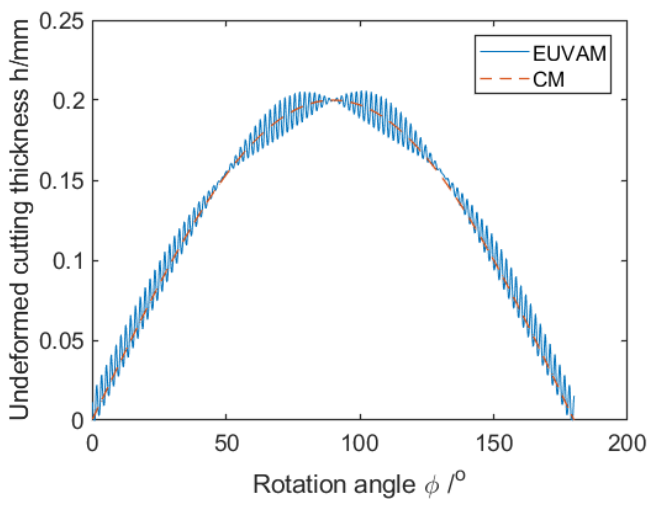

b. $n=6000 \mathrm{r} / \mathrm{min}$

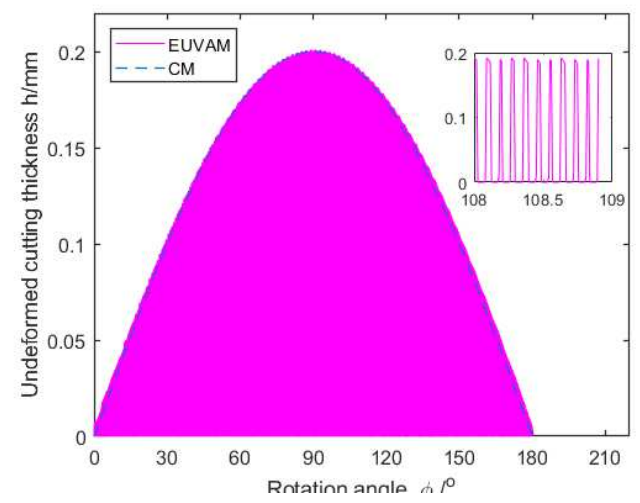

d. $n=300 \mathrm{r} / \mathrm{min}$

Fig. 9 Comparison of undeformed cutting thickness between EUVAM and CM under different spindle speed

$$
\text { ( } a=0.012 \mathrm{~mm}, b=0.008 \mathrm{~mm}, f=20000 \mathrm{~Hz}, R=4 \mathrm{~mm}, f_{t}=0.2 \mathrm{~mm} / \text { tooth }, N=2 \text { ) }
$$

\section{Cutting force modeling for EUVAM}

In order to reveal the detailed process of cutting force modeling for the elliptical ultrasonic vibration milling process, this paper focuses on cylindrical end mill. The modeling parameters include tool parameters: radius $R$, number of teeth $N$, helix angle $\beta$, pitch angle $\phi_{\mathrm{p}}$; the process parameters: spindle speed $n$, federate per tooth $f_{t}$, axial depth of cut $a_{p}$, radial depth of cut $a_{e}$; vibration parameters: ultrasonic frequency $f$, ultrasonic amplitude $a$ and $b$.

It is found that there is no essential difference between EUVAM and CM in the cutting mechanism and cutting force distribution along the cutting tooth, except that the former adds additional smallamplitude high-frequency elliptical vibration. The workpiece material is extruded on the rake face, resulting in elastic-plastic deformation and shear slip, and the cutting layer metal is separated from the matrix to form chips; the flank of the cutting tool scrapes on the machined surface, resulting in tissue fibrosis and work hardening. Therefore, the classical instantaneous rigid force model is also suitable for EUVAM. Refer to the mechanistic cutting force model for CM, the cutting edge is discretized into $m$ disks with thickness $\mathrm{d} z$ along its axial direction, and the cutting force elements acting on tooth $j$-th, cutting edge element $l$-th in the tangential, radial and axial direction are expressed as:

$$
\begin{aligned}
\mathrm{d} F_{t j l} & =g\left(\phi_{j l}\right)\left(K_{t c} h\left(\phi_{j l}\right)+K_{t e}\right) \mathrm{d} z \\
\mathrm{~d} F_{r j l} & =g\left(\phi_{j l}\right)\left(K_{r c} h\left(\phi_{j l}\right)+K_{r e}\right) \mathrm{d} z \\
\mathrm{~d} F_{a j l} & =g\left(\phi_{j l}\right)\left(K_{a c} h\left(\phi_{j l}\right)+K_{a e}\right) \mathrm{d} z
\end{aligned}
$$

where $h$ is the instantaneous undeformed cutting thickness of the cutting-edge element, which can be obtained from the method proposed in the above section; $K_{\mathrm{tc}}, K_{\mathrm{rc}}, K_{\mathrm{ac}}$ are the tangential, radial and axial specific cutting force; $K_{\mathrm{te}}, K_{\mathrm{re}}, K_{\mathrm{ae}}$ are the tangential, radial and axial edge force coefficients. $\mathrm{g}\left(\phi_{j l}\right)$ is the 
step function used to indicate whether the current cutting-edge element participates in cutting or not. Different from the cutting force model of conventional milling, when calculating the undeformed cutting thickness of EUVAM in the above section, whether the cutting tool is separated from the workpiece has been considered. Therefore, we can make $\mathrm{g}\left(\phi_{j l}\right)=1$.

As shown in Fig. 8, due to the existence of a helix angle, a point on the cutting edge will lag behind a point on the tooltip. The lag angle of a point at $z$ height in the axial direction can be expressed as:

$$
\psi=z \tan \beta / R
$$

For a cutting tool with uniformly distributed cutting teeth, the pitch angle $\phi_{\mathrm{p}}=2 \pi / N$. Suppose the rotation angle at the tooltip of the first tooth is $\phi_{10}$, then the rotation angle of the $l$-th cutting-edge element located on the $j$-th tooth can be expressed as:

$$
\phi_{j l}=\phi_{10}+(j-1) \phi_{p}+l \mathrm{~d} z \tan \beta / R
$$

Through coordinate transformation, the cutting force component in the machine tool coordinate system (Cartesian coordinate system) can be obtained as follows:

$$
\begin{array}{r}
\mathrm{d} F_{x j l}=-\mathrm{d} F_{t j l} \cos \left(\phi_{j l}\right)-\mathrm{d} F_{r j l} \sin \left(\phi_{j l}\right) \\
\mathrm{d} F_{y j l}=\mathrm{d} F_{t j l} \sin \left(\phi_{j l}\right)-\mathrm{d} F_{r j l} \cos \left(\phi_{j l}\right) \\
\mathrm{d} F_{z j l}=\quad \mathrm{d} F_{a j l}
\end{array}
$$

Integrate the cutting force acting on the cutting-edge element along the axial direction, and sum the cutting force for each tooth to yield the three-dimensional cutting forces as follows:

$$
\begin{gathered}
F_{x}=\sum_{j=1}^{N} \sum_{l=1}^{n} \mathrm{~d} F_{x j l} \\
F_{y}=\sum_{j=1}^{N} \sum_{l=1}^{n} \mathrm{~d} F_{y j l} \\
F_{z}=\sum_{j=1}^{N} \sum_{l=1}^{n} \mathrm{~d} F_{z j l}
\end{gathered}
$$

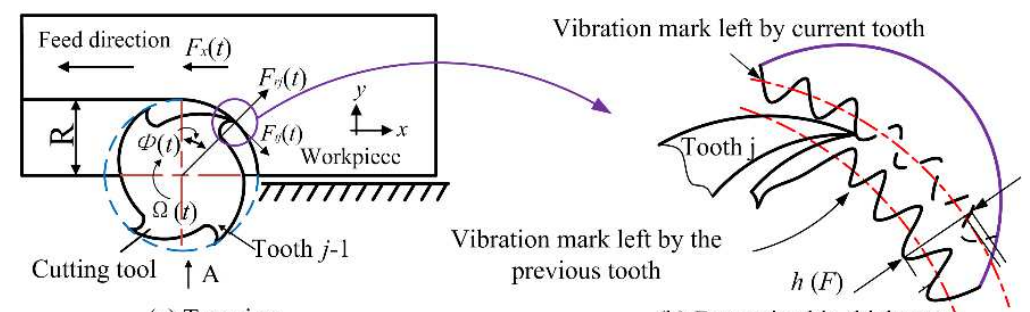

(a) Top view

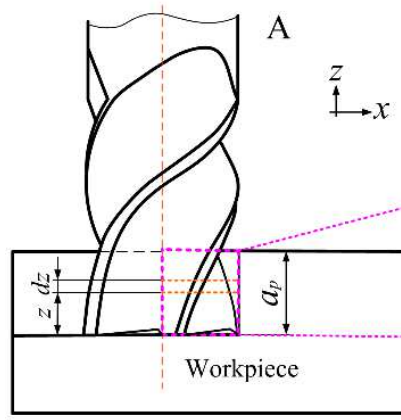

(c) Front view (b) Dynamic chip thickness '

(b)

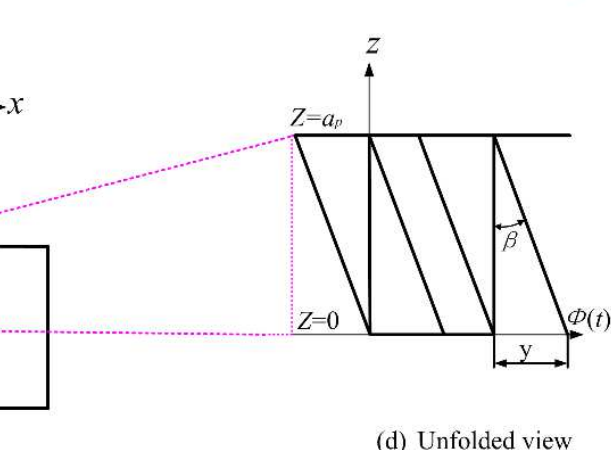

(d) Unfolded view

Fig.10 Cutting edge geometry and cutting force distribution in EUVAM

\section{Experimental verification and analysis}

Cutting force verification tests of EUVAM are carried out on a 5-axis vertical machining center Rambaudi Ramatic-1201g, and the experimental devices are shown in Fig.11 [19]. The tool holder used for EUVAM is composed of a tool handle, an elliptical piezoelectric transducer and a $12 \mathrm{~mm}$ diameter four-tooth cemented carbide end mill. The device can produce two-dimensional high-frequency vibrations in two directions perpendicular to each other, and its vibration frequency is about $17880 \mathrm{~Hz}$. 
The material of the test piece is titanium alloy Ti-6Al-4V, and its size is $200 \mathrm{~mm} \times 120 \mathrm{~mm} \times 60 \mathrm{~mm}$. The cutting force measuring devices adopts a three-way dynamometer Kistler9257b, the charge amplifier is Kistler5017a, and the cutting force measuring software is DynoWare signal analyzer, and the sampling frequency is set to $10 \mathrm{kHz}$.

The cutting force coefficient of titanium alloy Ti-6Al-4V obtained through the cutting force coefficient identification experiment is shown in Table 1 . The cutting parameters used in the verification tests are spindle speed $n=600 \mathrm{r} / \mathrm{min}$, feed per tooth $f_{\mathrm{t}}=0.20 \mathrm{~mm}$, radial depth of cut $a_{\mathrm{e}}=0.2 \mathrm{~mm}$ and axial cutting depth $a_{\mathrm{p}}=5.0 \mathrm{~mm}$; the elliptical vibration parameters are the amplitude in feed direction $a$ $=0.012 \mathrm{~mm}$, the amplitude normal to feed direction $b=0.008 \mathrm{~mm}$, and the vibration frequency $f=17880$ Hz. The comparison between the measured and predicted cutting forces in $X$ and $Y$ directions is shown in Fig.12. The figure shows that the predicted cutting force in $X$ direction is in good agreement with the measured one both in amplitude and shape, and the predicted cutting force in $Y$ direction is also in good agreement with the measured one in shape, except for some differences in amplitude. The reason is that the cutting force amplitude in the $Y$ direction is small due to the small radial depth of the cut used in the test. Other unknown small disturbances may cause a great difference in the amplitude ratio, but the absolute difference is not large. The experimental results show that the cutting force prediction model of EUVAM proposed in this chapter has significant prediction accuracy.

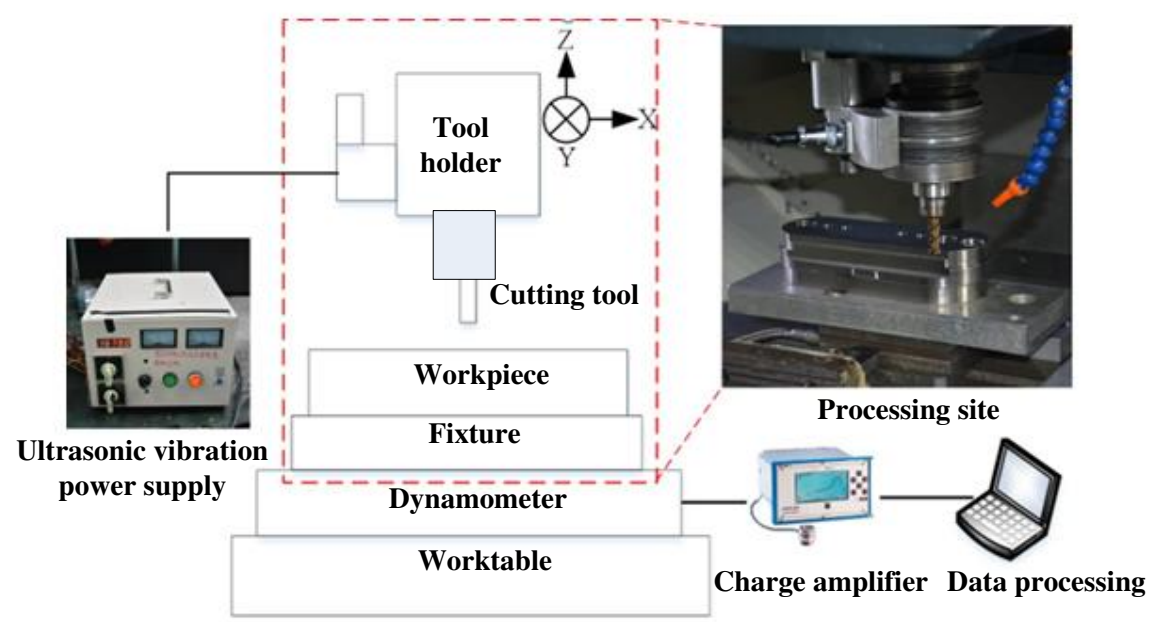

Fig.11 Schematic diagram of the experimental setup

Table 1 cutting force coefficients for material Ti-6Al-4V

\begin{tabular}{cccccc}
\hline$K_{\mathrm{tc}}(\mathrm{MPa})$ & $K_{\mathrm{rc}}(\mathrm{MPa})$ & $K_{\mathrm{ac}}(\mathrm{MPa})$ & $K_{\mathrm{te}}(\mathrm{MPa})$ & $K_{\mathrm{re}}(\mathrm{MPa})$ & $K_{\mathrm{ae}}(\mathrm{MPa})$ \\
\hline 2315 & 383.2 & -522.78 & 32.72 & 55.87 & -10.17 \\
\hline
\end{tabular}




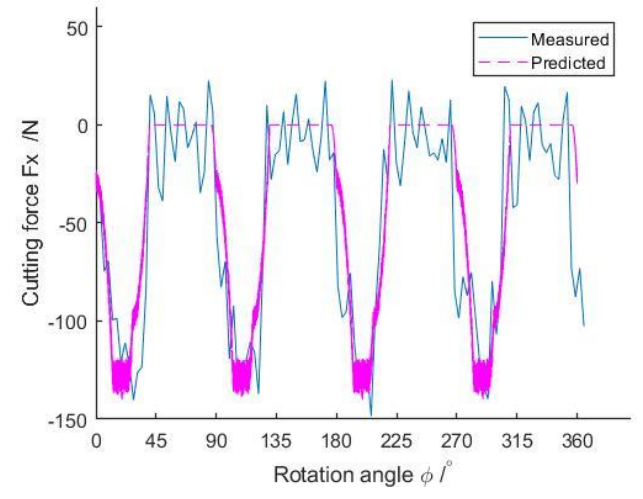

a. $F_{x}$

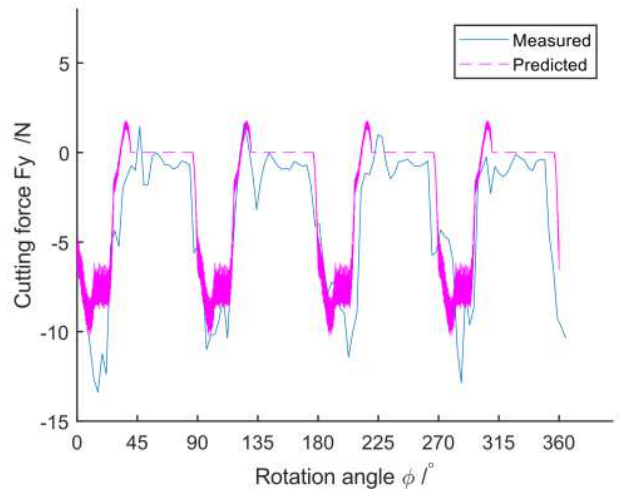

b. $F_{y}$

Fig.12 Comparison of measured cutting force and predicted one in EUVAM $\left(n=600 \mathrm{r} / \mathrm{min}, f_{\mathrm{t}}=0.20 \mathrm{~mm}, a_{\mathrm{e}}=0.2 \mathrm{~mm}, a_{\mathrm{p}}=5.0 \mathrm{~mm}, a=0.012 \mathrm{~mm}, b=0.008 \mathrm{~mm}, f=17880 \mathrm{~Hz}\right)$

In order to further reveal the effect of the proportion of empty cutting time in EUVAM, that is, the spindle speed on cutting force, the cutting forces in EUVAM and CM under different spindle speeds are predicted, and the comparison is shown in Fig. 13. The figure shows that with the decrease of spindle speed, that is, the elliptical vibration speed is dominant and the proportion of empty cutting time increases, the cutting force of EUVAM in both $X$ direction and $Y$ direction shows a significant decrease. As the spindle speed $n=900 \mathrm{r} / \mathrm{min}$, there is little difference in the cutting force between EUVAM and CM; however, as the spindle speed $n=300 \mathrm{r} / \mathrm{min}$, the cutting force in EUVAM decreases by nearly $50 \%$ compared with CM in both $X$ direction and $Y$ direction. Fig. 14 shows the comparison of the measured cutting force in $X$ and $Y$ directions between EUVAM and CM when the spindle speed $n=600 \mathrm{r} / \mathrm{min}$. The figure shows that EUVAM can significantly reduce the cutting force compared to CM.
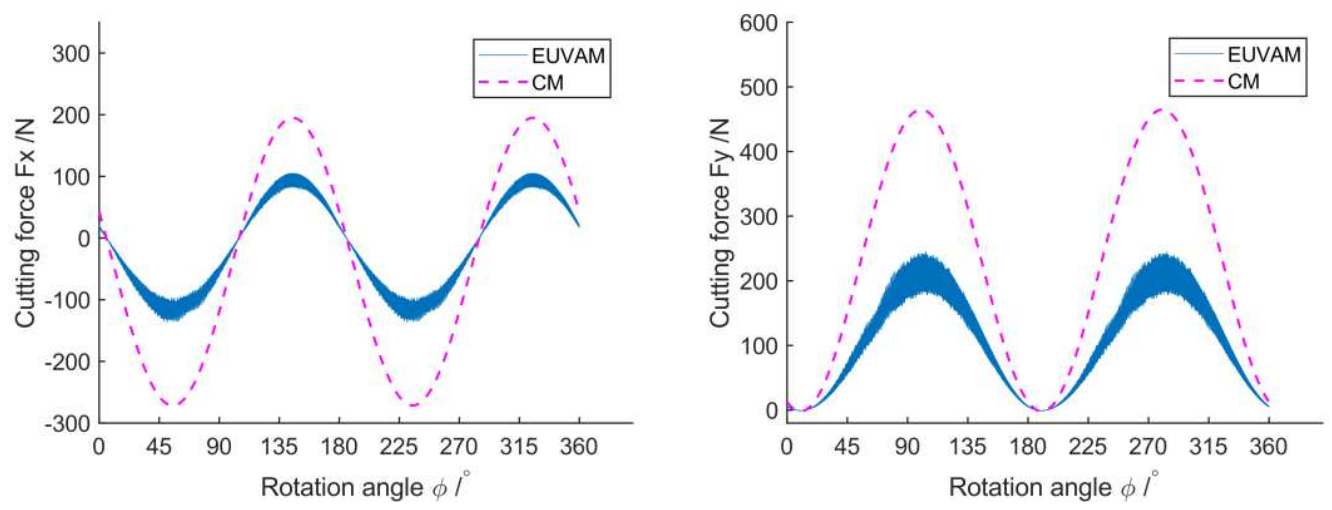

a. $n=300 \mathrm{r} / \mathrm{min}$
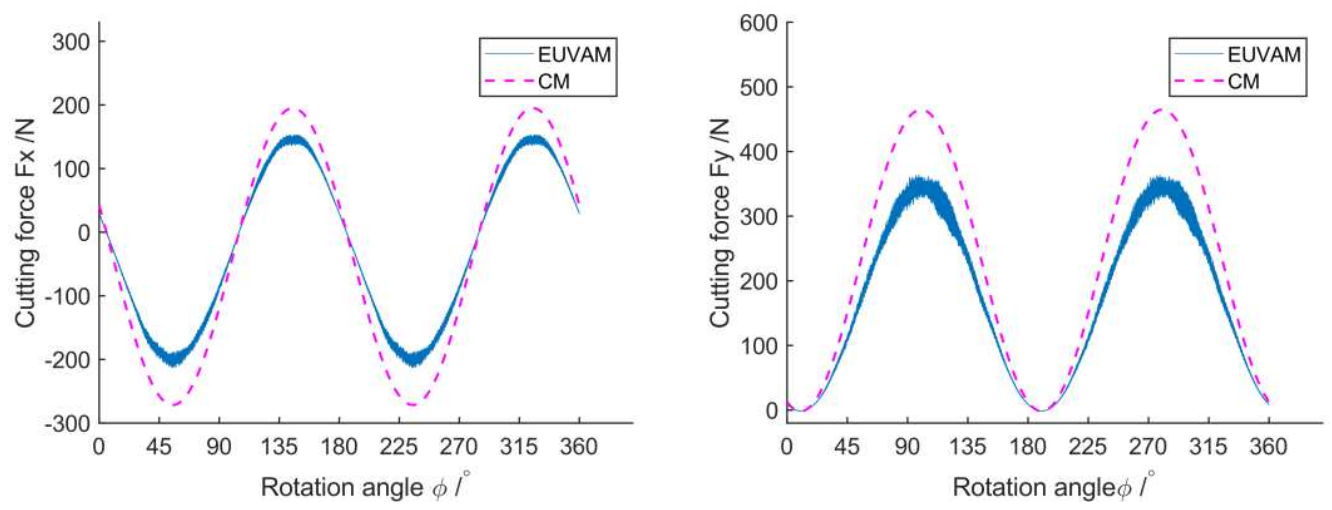

b. $n=600 \mathrm{r} / \mathrm{min}$ 

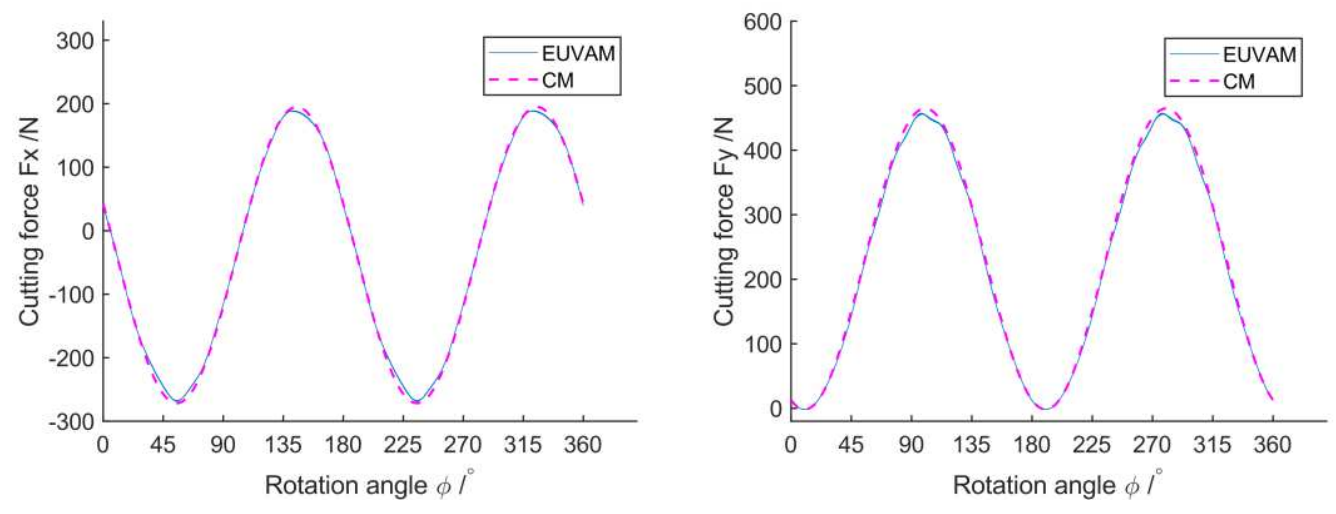

c. $\quad n=900 \mathrm{r} / \mathrm{min}$

Fig.13 Comparison of predicted cutting forces between EUVAM and CM $\left(f_{\mathrm{t}}=0.10 \mathrm{~mm}, a_{\mathrm{e}}=12.0 \mathrm{~mm}, a_{\mathrm{p}}=2.0 \mathrm{~mm}, a=0.012 \mathrm{~mm}, b=0.008 \mathrm{~mm}, f=17880 \mathrm{~Hz}\right)$

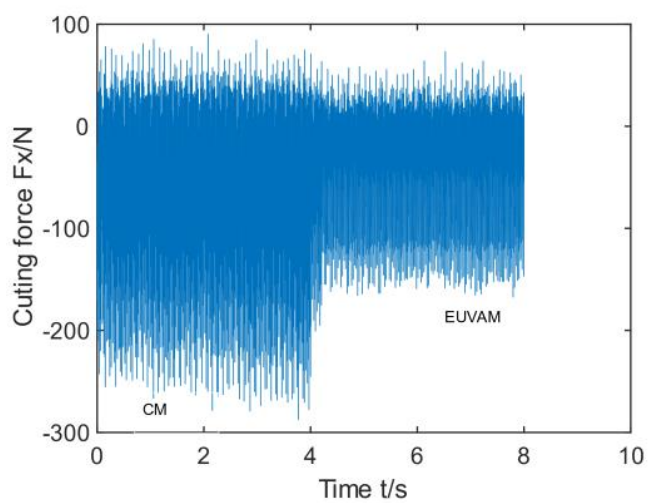

a. $F_{x}$

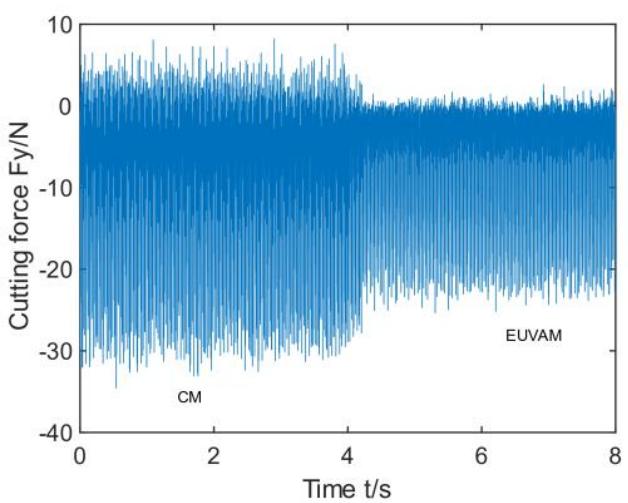

b. $F_{y}$

Fig. 14 Comparison of measured cutting force between EUVAM and CM

$\left(n=600 \mathrm{r} / \mathrm{min}, f_{\mathrm{t}}=0.20 \mathrm{~mm}, a_{\mathrm{e}}=0.2 \mathrm{~mm}, a_{\mathrm{p}}=5.0 \mathrm{~mm}, a=0.012 \mathrm{~mm}, b=0.008 \mathrm{~mm}, f=17880 \mathrm{~Hz}\right)$

\section{Conclusions}

In this paper, the motion equations in EUVAM are first established, and on the basis, the ultrasonic milling force model is established combined with the undeformed cutting thickness in EUVAM with the use of the Z-map model of the workpiece. The proposed model is verified by cutting tests, and the influence of cutting conditions on both the undeformed cutting thickness and the ultrasonic milling force is analyzed.

(1) Due to the existence of ultrasonic vibration, the actual cutting speed in EUVAM is much higher than that in CM under the same cutting parameters. Therefore, the adoption of EUVAM can realize highspeed milling to some extent at a relatively low spindle speed.

(2) The adoption of the Z-map model can greatly simplify the calculation complexity of undeformed cutting thickness in EUVAM. The ratio of cutting-edge rotation linear velocity to ultrasonic vibration linear velocity, namely, the proportion of idle cutting time, determines the intermittent degree of EUVAM. An appropriate proportion of idle cutting time is helpful to improve the tool life and the surface quality of the workpiece.

(3) According to the comparison between the predicted cutting force and the experimental one, the cutting force prediction method proposed in this paper can accurately predict the cutting force in EUVAM. Simulation results show that, with the increase of the proportion of idle cutting time, the cutting force of EUVAM in both $X$ direction and $Y$ direction shows a significant decrease, and compared with 
the conventional milling, the maximal cutting force can be reduced by up to $50 \%$.

\section{Funding}

This research is financially supported by the natural science foundation of Hunan Province (2020JJ4270)

\section{Conflicts of interest}

No conflict of interest exists

\section{Availability of data and material}

The data used to support the findings of this study are included within the article

\section{Code availability}

Not applicable

\section{Ethics approval}

Not applicable

\section{Consent to participate}

Not applicable

\section{Consent to publication}

Not applicable

\section{Reference}

[1] Jin X, Xie B. Experimental study on surface generation in vibration-assisted micro-milling of glass. Int $J A d v$ Manuf Technol 2015, 81(1-4): 507-512

[2] Shamoto E, Moriwaki T Ultraprecision Diamond Cutting of Hardened Steel by Applying Elliptical Vibration Cutting. CIRP Annals 1999, 48(1): 441-444

[3] $\mathrm{Xu} \mathrm{W}$, Zhang L, Wu Y. Elliptic vibration-assisted cutting of fiber-reinforced polymer composites: understanding the material removal mechanisms. Compos Sci Technol 2014, 92: 103-111.

[4] Sui H, Zhang X, Zhang D, Jiang X, Wu R. Feasibility study of high-speed ultrasonic vibration cutting titanium alloy. J Mater Process Technol 2017, 247(19): 111-120.

[5] Insperger T, Lehotzky D, Stepan G. Regenerative delay parametric forcing and machine tool chatter: A review. IFAC-PapersOnLine 2015, 48(12): 322-327.

[6] Altintas Y, Weck M. Chatter stability of metal cutting and grinding. CIRP Annals 2004, 53(2): 619-642.

[7] Xiao M, Karube S, Soutome T, Sato K. Analysis of chatter suppression in vibration cutting. Int J Mach Tools Manuf 2002, 42(15): 1677-1685.

[8] Ma C, Ma J, Shamoto E, Moriwaki T. Analysis of regenerative chatter suppression with adding the ultrasonic elliptical vibration on the cutting tool. Precis Eng 2011, 35(2): 329-338.

[9] Wan S, Jin X, Maroju NK, Hong J. Effect of vibration assistance on chatter stability in milling. Int J Mach Tools Manuf 2019, 145: 103432.

[10] Gao J, Altintas Y. Chatter stability of synchronized elliptical vibration assisted milling. CIRP J Manuf Sci Technol 2020, 28:76-86

[11] Muhammad R, Ahmed N, Deiral M, Roy A, Silberschmidt VV. Computational study of ultrasonically-assisted turning of Ti alloys. Adv Mat Res 2011, 223: 30-36

[12] Tong J, Wei G. Characteristics of cutting force during titanium alloy processed with UEVC. J Shock Vib 2019, 38 (9): $208-214$

[13] Liu J, Jiang X, Zhang G, Zhang M, Zhang D. Investigation of the Effect of Vibration Amplitude on the Surface 
Integrity in High-speed Rotary Ultrasonic Elliptical Machining for Side Milling of Ti-6Al-4V. J Mech Eng. 2019, 55(11): 215-223

[14] Abootorabi Z, Razfar M, Abdullah A. Investigation of the effect of cutting speed and vibration amplitude on cutting forces in ultrasonic-assisted milling. Proc Inst Mech Eng B J Eng Manuf 2012, 226(7):1185-1191

[15] Han X, Zhang D. Effects of separating characteristics in ultrasonic elliptical vibration-assisted milling on cutting force, chip, and surface morphologies. Int J Adv Manuf Technol 2020, 108: 3075-3084

[16] Li Z, Zhu L, Yang Z, Ma J, Cao W. Investigation of tool-workpiece contact rate and milling force in elliptical ultrasonic vibration assisted milling. Int J Adv Manuf Technol 2021, https://doi.org/10.1007/s00170-02107946-y

[17] Li Z, Liu Q. Surface topography and roughness in hole-making by helical milling. Int J Adv Manuf Technol 2013, 66(9-12):1415-1425.

[18] Lee SK, Ko SL. Development of simulation system for machining process using enhanced Z map model. $J$ Mater Process Technol 2002, 130(1):608-617.

[19] Gradisek J, Kalveram M, Weinert K. Mechanistic identification of specific force coefficients for a general end mill. Int J Mach Tool Manuf, 2004, 44(4):401-414 